\title{
IS CONDITIONING REALLY INCOMPATIBLE WITH HOLISM?
}

\section{CARL WAGNER}

Abstract. Jonathan Weisberg claims that certain probability assessments constructed by Jeffrey conditioning resist subsequent revision by a certain type of after-the-fact defeater of the reasons supporting those assessments, and that such conditioning is thus "inherently anti-holistic." His analysis founders, however, in applying Jeffrey conditioning to a partition for which an essential rigidity condition clearly fails. Applied to an appropriate partition, Jeffrey conditioning is amenable to revision by the sort of after-the-fact defeaters considered by Weisberg in precisely the way that he demands.

Key words: defeater, holism, Jeffrey conditioning, rigidity.

1. Holism Denied. Confirmational holism requires that a belief's empirical justification be sensitive to background belief. For Bayesian epistemology this entails among other things that your probability assessments be amenable to revision in response to the discovery of after-thefact defeaters of reasons supporting those assessments. Jonathan Weisberg (2009) thinks that a certain class of probability assessments constructed by Jeffrey conditioning resist such revision, and that such conditioning is thus "inherently anti-holistic." Typifying such cases is, he claims, the following example: Suppose that $E$ asserts that a certain jelly bean is red, and $F$ asserts that the lighting under which you are to view this jelly bean is contrived to make it appear red, whether it is or not. Let $p$ be your prior probability measure on the algebra generated by $E$ and $F$, and suppose that

$$
0<p(E), p(F)<1 \text {. }
$$

One can easily imagine scenarios in which

$$
p(E F)=p(E) p(F)
$$

and Weisberg postulates this independence condition. Suppose now that you have the opportunity to view the jelly bean, which results in your having the non-doxastic experience "as of red," denoted $\mathcal{E}$. This experience is assumed to increase your confidence in $E$, though perhaps not rendering $E$ certain for you. How should you revise $p$ in the light of this experience?

Weisberg has you revise $p$ by Jeffrey conditioning on the partition $\{E, \bar{E}\}$,

thereby updating $p$ to the probability measure $q$ given by

$$
q(\cdot)=p(\cdot \mid E) q(E)+p(\cdot \mid \bar{E}) q(\bar{E})
$$

where 


$$
q(E)>p(E) .
$$

You now learn that the contrived lighting was in effect during your viewing, and so you revise $\mathrm{q}$ to $r$ by strict conditioning on $F$. Since $F$ is an after-the-fact defeater of your non-doxastic reason $\mathcal{E}$ for becoming more confident in $E$, one would hope that

$$
q(E \mid F)=p(E)
$$

or, at the very least, that

$$
q(E \mid F)<q(E)
$$

But this cannot be the case, for, as Weisberg notes, it follows from (3) that

(7) $q(F \mid E)=p(F \mid E)$, and

(8) $q(F \mid \bar{E})=p(F \mid \bar{E})$,

and conditions (2), (7), and (8) imply that

(9) $q(E \mid F)=q(E)$.

In short, the rigidity conditions (7) and (8) preserve the $p$-independence of $E$ and $F$. Hence, updating the prior $p$ by Jeffrey conditioning on the partition $\{E, \bar{E}\}$ creates a posterior $q$ not amenable to correction in response to the discovery of the undercutting defeater $F$. Jeffrey conditioning is thus "inherently anti-holistic" (Weisberg 2009, p. 808).

2. Parochialism. But wait. Updating $p$ to $q$ by Jeffrey conditioning on the partition $\{E, \bar{E}\}$ is justified if and only if (I) based on the total evidence, old as well as new, one judges it appropriate to revise one's prior probability $p(E)$ to the new value $q(E)$, and (II) one has learned nothing new about the relevance of $E$, or of $\bar{E}$, to any other event, and thus judges it appropriate for each event $A$ to maintain the values of the conditional probabilities $p(A \mid E)$ and $p(A \mid \bar{E})$ in the posterior $q$. In the present case, where $A$ is an element of the algebra generated by $E$ and $F$, this condition is easily seen to be equivalent simply to the conjunction of conditions (7) and (8). Now (7) is perfectly reasonable (why should learning that the jelly bean is red prompt you to revise your probability that the contrived lighting was in effect?). But (8) is completely unreasonable. After all, if you've experienced $\mathcal{E}$, and have subsequently learned that the jelly bean in question is not red, shouldn't you become much more confident that the contrived lighting was in effect during your viewing, ${ }^{1}$ maybe even to the extent of setting $q(F \mid \bar{E})=1$ ? And doesn't this observation, and the fact that it rules out updating $p$ by Jeffrey conditioning on $\{E, \bar{E}\}$, vitiate Weisberg's example? 
Since one not infrequently encounters confusion on this score, it may be worth pointing out that Jeffrey has always emphasized that one must attend to both (I) and (II) in deciding whether it is appropriate to update $p$ by formula (3). ${ }^{2}$ Indeed he has labeled the error of neglecting to consider criterion (II) with the opprobrium parochialism (Jeffrey 1988, p. 122). ${ }^{3}$ That Weisberg has fallen victim to parochialism is evident from his gloss of Jeffrey's rule (Weisberg 2009, p. 801):

Jeffrey Conditionalization. When experience directly changes your credences over a partition $\left\{E_{i}\right\}$ from $p\left(E_{i}\right)$ to $q\left(E_{i}\right)$, set your new credences to $q(\cdot)=\sum_{i} p\left(\cdot \mid E_{i}\right) q\left(E_{i}\right)$.

Weisberg may protest that, on the contrary, he is no fan of rigidity, and that he has made this clear in the statement (Weisberg 2009, p. 807), "We can gloss this point as follows: rigidity implies the absence of after-the-fact, undercutting defeaters for non-doxastic reasons." But there is a difference between disliking rigidity because of its consequences, and declining to embrace it on prima facie grounds of unreasonableness. Of course Weisberg understands that (3) implies both (7) and (8). But his pragmatic understanding of this relation is that once one has opted for (3) as an updating rule, (7) and (8) come along as consequences, whether one likes it or not. This gets things exactly backwards, for judging that (7) and (8) are reasonable is a precondition for employing (3). Failing to grasp this fact renders one powerless to critique (7) or (8) on grounds independent of their consequences. And it leads Weisberg to think that he has discovered a serious defect in Jeffrey's rule. For, he argues, $p$ has been updated to $q$ by Jeffrey's rule in response to $\mathcal{E}$, with the result that $q$ cannot be repaired by conditioning in response to the discovery that $F$. And since, he claims, Jeffrey's rule fails to provide any guidance regarding the choice of the partition on which to update, Jeffrey conditionalizers are stuck with updating $p$ on the partition $\{E, \bar{E}\}$, whether they like it or not. But this conflates two issues. It is certainly true that Jeffrey has specified no procedure for picking out a uniquely rational partition on which to update. ${ }^{5}$ But he has furnished grounds for rejecting the application of Jeffrey updating to any particular partition, namely, the considered judgment that rigidity is unwarranted for that partition. To demand more than that is to fundamentally misunderstand the role that Jeffrey intended his rule to play in probability revision.

3. Holism Attained. If it is unreasonable to update $p$ to $q$ by formula (3) in response to the experience $\mathscr{E}$, is there a different partition to which Jeffrey's rule could be applied, and which would yield a posterior amenable to correction by conditioning in light of the discovery that $\mathrm{F}$ ? Consider the partition $\{E \cup F, \bar{E} \cap \bar{F}\}$. Since either $E$ or $F$ makes it more likely that you will experience $\mathscr{E}$, having that experience should lead you to revise $p(E \cup F)$ to (let us call it) $q^{*}(E \cup F)$, where 


$$
q^{*}(E \cup F)>p(E \cup F) .
$$

Indeed, if you have good reason to believe that you could not have experienced $\mathscr{E}$ unless the jelly bean were red or the lighting contrived, you might go so far as to set $q^{*}(E \cup F)=1$. But let us suppose that the viewing scenario is such that you might experience $\mathscr{E}$ even when viewing a nonred jelly bean under non-contrived lighting. Perhaps, for example, the lighting is dim, though not tinted red, and there are purple jelly beans among those from which the one you viewed was selected. Then, along with (10), you might reasonably set

$$
q^{*}(\bar{E} \cap \bar{F})>0 .
$$

If (as would be reasonable under many ways of elaborating the scenario under consideration) nothing about $\mathscr{E}$ would prompt you to alter the prior odds between any of the three ways (viz., $E \cap F, \bar{E} \cap F$, or $E \cap \bar{F}$ ) in which $E \cup F$ might materialize, you would then be justified ${ }^{6}$ in extending $q^{*}$ to the entire algebra A generated by $E$ and $F$ in accord with Jeffrey's Rule :

$$
\text { For all } A \in \mathrm{A}, \quad q^{*}(A)=q^{*}(E \cup F) p(A \mid E \cup F)+q^{*}(\bar{E} \cap \bar{F}) p(A \mid \bar{E} \cap \bar{F}) .
$$

THEOREM 1. The propositions $E$ and $F$ are $q^{*}$ - negatively relevant to each other.

Proof. By (12) and (10), $q^{*}(E)=q^{*}(E \cup F) p(E) / p(E \cup F)>p(E)$. By (12) and (2), $q^{*}(E \mid F)=q^{*}(E \cap F) / q^{*}(F)=p(E \cap F) / p(F)=p(E)$.

If we were to follow Weisberg's development here, we would assume that you now discover that the contrived lighting was in effect during your viewing. But we might as well treat the more general case in which you simply become more confident in $F$, and in such a way that you are not inclined to change the $q^{*}$-odds between $E \cap F$ and $\bar{E} \cap F$, or between $E \cap \bar{F}$ and $\bar{E} \cap \bar{F}$. You would then be justified in updating $q^{*}$ to (let us call it) $r^{*}$ by Jeffrey conditioning on the partition $\{F, \bar{F}\}$, where

$$
r^{*}(F)>q^{*}(F) .
$$

THEOREM 2. The revision $r^{*}$ satisfies the minimally acceptable defeater inequality $r^{*}(E)<q^{*}(E)$. Moreover, $r^{*}(E)=p(E)$ if and only if $r^{*}(F)=1$.

Proof. By the definition of $r^{*}$ and the total probability law,

$$
r^{*}(E)-q^{*}(E)=\left[r^{*}(F)-q^{*}(F)\right] \times\left[q^{*}(E \mid F)-q^{*}(E \mid \bar{F})\right]<0,
$$


the first factor on the right hand side of (14) being positive by (13), and the second factor being negative by Theorem 1. If $r^{*}(F)=1$, then $r^{*}(E)=q^{*}(E \mid F)=p(E)$, as shown in the proof of

Theorem 1. Suppose that $r^{*}(E)=p(E)$. Then, since $r^{*}$ comes from $q^{*}$ by Jeffrey conditioning on $\{F, \bar{F}\}, q^{*}(E \mid F)=p(E)=r^{*}(E)=r^{*}(F) q^{*}(E \mid F)+r^{*}(\bar{F}) q^{*}(E \mid \bar{F})$ and so $r^{*}(\bar{F})\left[q^{*}(E \mid F)-q^{*}(E \mid \bar{F})\right]=0$. Since $q^{*}(E \mid F)-q^{*}(E \mid \bar{F}) \neq 0$ by Theorem 1 , it follows that $r^{*}(\bar{F})=0$, whence $r^{*}(F)=1$.

It is easy to see that if $q^{*}$ comes from $p$ by strict conditioning on $E \cup F$, rather than by Jeffrey conditioning on $\{E \cup F, \bar{E} \cap \bar{F}\}$, then Theorems 1 and 2 continue to hold. Thus either sort of conditioning results in a posterior $q^{*}$ amenable to revision based on an after-the-fact increase in confidence in the defeater $F$, and with exactly the properties that Weisberg demands. And the probability $q^{*}(E)$ reverts all the way back to $p(E)$ in just those scenarios in which you attain after-the-fact certainty regarding the defeater $F$.

\section{Notes}

1. Although it is obvious that it should be the case that $q(F \mid \bar{E})>p(F \mid \bar{E})$, the following heuristic argument also highlights this inequality: Condition (2) holds by design, condition (7) is, as we have argued, unexceptionable, and condition (6) expresses the minimally acceptable effect of discovering the defeater $F$. But (2), (6), and (7) hold only if $q(F \mid \bar{E})>p(F \mid \bar{E})$. For by (6), $E$ and $F$ are $q$-negatively relevant to each other, and so $\bar{E}$ and $F$ are $q$-positively relevant to each other. So $q(F \mid \bar{E})>q(F \mid E)=p(F \mid E)=p(F \mid \bar{E})$, , the latter two equalities following, respectively, from (7) and (2).

2. In The Logic of Decision (1965, section 11.3) Jeffrey expresses condition (I) by saying that "the passage of experience has led the agent to change his degree of belief in" $E$ from $p(E)$ to $q(E)$, while expressing condition (II) by saying that the change from $p$ to $q$ "originated in" $E$. The latter terminology is unfortunate in perhaps not conveying in the most memorable way that condition (II) is a prerequisite for applying the updating formula (3), and not simply a consequence of applying that formula. But in the illustrative example ("The mudrunner") immediately following these remarks, it is clear that condition (II) is being checked for reasonableness prior to applying (3), for Jeffrey says "However, the forecast should have no effect on his degrees of belief in the proposition that the horse will win conditionally on the course being muddy, or on its not being muddy." In any case, in later publications ((Jeffrey 1970, p.173), where condition (II) is termed "rigidity"; (Jeffrey 1988, p.124), where (II) is termed "sufficiency"; and (Jeffrey 2004, pp. 53-54), where (II) is termed "invariance"), Jeffrey states in 
unambiguous terms that one must judge that condition (II) is satisfied before applying formula (3). In the mudrunner example this judgment occurs almost effortlessly. In other cases, however, one may need to think long and hard about whether rigidity is reasonably assumed. One can, of course, overlook some subtlety and judge wrongly in this regard. But a conscious effort to judge whether it is reasonable to embrace rigidity needs to be made before one decides to employ the updating formula (3).

3. Jeffrey actually applies the term parochialism to the error of updating $p$ to $q$ by strict conditioning on $E$ without considering whether the invariance condition $q(A \mid E)=p(A \mid E)$ is reasonably assumed for all events $A$ in the relevant algebra. But it is implicit that the term is also applicable to the error of updating on a partition without checking condition (II).

4. I suppose it is conceivable that Weisberg intends the phrase "experience directly changes your credences" to mean not simply that you have come to assign new probabilities to the events $E_{i}$, but that you have given conscious consideration to whether anything in that experience warrants disturbing any of the prior conditional probabilities $p\left(A \mid E_{i}\right)$ in the process of updating $p$, and that you have judged that this is not the case. Then, while not being guilty of parochialism, he has, while fully cognizant of criterion (II) for implementing Jeffrey's rule, neglected to notice that it is clearly not reasonable to expect the rigidity condition $q(F \mid \bar{E})=p(F \mid \bar{E})$ to hold.

5. Weisberg claims (p.802) that, absent such a specification, "Jeffrey Conditionalization is actually vacuous. Unless we say what partition is directly affected by experience, and what values it ought to get, we can do any update we want without violating Jeffrey conditionalization." If "violating Jeffrey conditionalization" means "violating the conditions required to apply Jeffrey's rule," the latter statement false, as we have seen in the case of Weisberg's demonstrably unjustified application of Jeffrey's rule to the partition $\{E, \bar{E}\}$. But Weisberg seems to mean by this phrase simply "not using an updating formula having the form $q(\cdot)=\sum_{i} p\left(\cdot \mid E_{i}\right) q\left(E_{i}\right)$." To term this a "violation" of Jeffrey's rule seems very strange. After all, Jeffrey never claimed that his rule was the be-all-and-end-all of probability revision. Indeed, he devised an entirely different method of probability revision (Jeffrey, 1991, 1995) called reparation as a solution to one version of the old evidence problem. Beyond that, Weisberg fundamentally misunderstands the role that Jeffrey conditioning is intended to play in probability revision. Suppose that based on my total experience, old as well as new, I come to revise my prior probabilities $p\left(E_{i}\right)$ of the events in some partition $\left\{E_{i}\right\}$ to the new values $q\left(E_{i}\right)$. And suppose that I judge that, for each event $A$ in the relevant algebra, nothing in that experience should prompt me to revise any of the conditional probabilities $p\left(A \mid E_{i}\right)$. I then proceed to update $p$ by Jeffrey's rule on $\left\{E_{i}\right\}$. Later, it occurs to me there is a partition $\left\{F_{j}\right\}$ coarser than $\left\{E_{i}\right\}$, in the sense each $E_{i}$ is a subset of some $F_{j}$, and that nothing in my experience should 
prompt me to revise any of the conditional probabilities $p\left(A \mid F_{j}\right)$. So I could have updated on the simpler partition $\left\{F_{j}\right\}$. This is not, as Weisberg seems to think, a scandalous state of affairs. In either case, I will arrive at exactly the same posterior $q$, the only difference being that my updating formula will be simpler in the latter case. As Diaconis and Zabell (1982) point out in their fundamental paper on Jeffrey conditioning, sometimes one must simply update $p$ to $q$ by what they call "total reassessment." If the set $\Omega$ of possible states of the world is countable, and $p\left(\omega_{i}\right)=0 \Rightarrow q\left(\omega_{i}\right)=0$, then such reassessment qualifies formally as a case of Jeffrey conditioning on the partition $\left\{\left\{\omega_{i}\right\}\right\}$. This also scandalizes Weisberg (2009, p. 802) who misses the point that Jeffrey conditioning, when applicable, is simply a labor-saving tool that cuts down on the number of events whose probabilities need to be reassessed. We may fail to make the most efficient use of this tool by updating on a finer partition than we need to, and we may overlook evidence that would alter certain conditional probabilities in such a way that revision by Jeffrey conditioning on a particular partition is inapplicable. But such oversights and mistakes are correctable. The important thing is, in the spirit of holism, to be alert to the possibility of their occurring.

6. This odds rigidity is equivalent to (and often more salient than) the more familiar conditional probability rigidity criterion for updating by Jeffrey conditioning. See Jeffrey (1992, p.125), noting that there is only one way for the event $\bar{E} \cap \bar{F}$ to materialize, since the algebra on which $p$ and $q^{*}$ are defined is assumed here to be simply the algebra generated by $E$ and $F$.

\section{References}

1. Diaconis, P. and S. Zabell (1982). Updating Subjective Probability, Journal of the American Statistical Association 77, 822-830.

2. Jeffrey, R. C. (1965). The Logic of Decision (New York: Mcgraw-Hill) ; second edition 1983 (Chicago: The University of Chicago Press).

3. (1970). Dracula Meets Wolfman: Acceptance vs. Partial Belief. (In M. Swain (ed.), Induction, Acceptance, and Rational Belief, Dordrecht: Reidel), 157-185.

4. (1988). Conditioning, Kinematics, and Exchangeability. (In B. Skyrms and W. Harper (eds.), Causation, Chance, and Credence, vol. 1. Dordrecht: Kluwer.).

5. (1991). Postscript 1991: New Explanation Revisited (in Jeffrey 1992.)

6. (1992). Probability and the Art of Judgment. (Cambridge: Cambridge University Press), 117-154. 
7. (1995). Probability Reparation: The Problem of New Explanation, Philosophical Studies 77, 97-102.

8. (2004). Subjective Probability: The Real Thing (Cambridge: Cambridge University Press).

9. Weisberg, J. (2009). Commutativity or holism? A dilemma for conditionalizers. British Journal for the Philosophy of Science 60, 793-812. 